Revue internationale P.M.E.

Économie et gestion de la petite et moyenne entreprise

\title{
L'entrepreneur comme clé de voûte de l'internationalisation rapide de son entreprise
}

\section{Mathieu Cabrol et Véronique Favre-Bonté}

Volume 24, numéro 2, 2011

URI : https://id.erudit.org/iderudit/1012687ar

DOI : https://doi.org/10.7202/1012687ar

Aller au sommaire du numéro

Éditeur(s)

Presses de l’Université du Québec

ISSN

0776-5436 (imprimé)

1918-9699 (numérique)

Découvrir la revue

Citer cet article

Cabrol, M. \& Favre-Bonté, V. (2011). L'entrepreneur comme clé de voûte de l'internationalisation rapide de son entreprise. Revue internationale P.M.E., 24(2), 111-137. https://doi.org/10.7202/1012687ar

\section{Résumé de l'article}

L'étude des entreprises nouvellement internationales (ENI) occupe une place de plus en plus importante dans la littérature en entrepreneuriat. Contrairement aux entreprises traditionnelles, ces ENI, dénommées aussi " born globals " (McKinsey \& Co., 1993) ou encore " international new ventures " (Oviatt et McDougall, 1994) se définissent comme des entreprises qui, dès leur création ou peu de temps après, cherchent à dériver un avantage concurrentiel de l'utilisation de ressources et de la vente de produits dans de multiples pays. Notre recherche vise à comprendre comment le réseau et les caractéristiques de l'entrepreneur interviennent dans l'internationalisation de jeunes entreprises. Nous avons étudié six cas d'entreprises qui se sont internationalisées dès la création ou peu de temps après. Les caractéristiques de l'entrepreneur, ses compétences, son expérience ainsi que son réseau personnel sont importants pour la création de son entreprise à l'international et pour nouer les premiers contacts sur les marchés étrangers. La personnalité et le réseau de l'entrepreneur agissent en interaction ; ils orientent tour à tour la création de l'entreprise et ses premiers pas sur les marchés étrangers.
Ce document est protégé par la loi sur le droit d'auteur. L'utilisation des services d’Érudit (y compris la reproduction) est assujettie à sa politique d'utilisation que vous pouvez consulter en ligne.

https://apropos.erudit.org/fr/usagers/politique-dutilisation/ 


\title{
L'entrepreneur comme clé de voûte de l'internationalisation rapide de son entreprise
}

\author{
Mathieu CABROL \\ GSCM Montpellier Business School \\ Véronique FAVRE-BONTÉ \\ Université de Savoie (IAE Savoie Mont-Blanc), IREGE
}

\section{MOTS CLÉS}

Internationalisation - Réseau - Caractéristiques de l'entrepreneur PME - Création d'entreprise

\begin{abstract}
LES AUTEURS
MATHIEU CABROL est professeur assistant au Groupe Sup de Co Montpellier (France). II est titulaire d'un doctorat en sciences de gestion. II a écrit plusieurs articles dans des revues académiques, notamment dans European Management Journal et International Journal of Entrepreneurship and Innovation. II a aussi publié plusieurs chapitres de livres au sujet de l'entrepreneuriat international. II s'est aussi occupé de l'accompagnement de jeunes entreprises technologiques et est spécialisé dans le domaine du développement international des jeunes entreprises. Courriel: <mathieucabrol@hotmail.com >.

VÉRONIQUE FAVRE-BONTÉ est maître de conférences à l'Université de Savoie où elle enseigne la stratégie, l'intelligence économique et la théorie des organisations. Elle est responsable d'un master 2 Commerce responsable à l'international. Ses recherches et publications sont centrées sur les stratégies internationales et l'innovation dans les services. Elle a écrit entre autres dans des revues telles que la RIPME et Management International. Courriel: <veronique.favre-bonte@univ-savoie.fr>.
\end{abstract}

\section{RÉSUMÉ}

L'étude des entreprises nouvellement internationales (ENI) occupe une place de plus en plus importante dans la littérature en entrepreneuriat. Contrairement aux entreprises traditionnelles, ces ENI, dénommées aussi «born globals» (McKinsey \& Co., 1993) ou encore « international new ventures » (Oviatt et McDougall, 1994) se définissent comme des entreprises qui, dès leur création ou peu de temps après, cherchent à dériver un avantage concurrentiel de l'utilisation de ressources et de 
la vente de produits dans de multiples pays. Notre recherche vise à comprendre comment le réseau et les caractéristiques de l'entrepreneur interviennent dans l'internationalisation de jeunes entreprises. Nous avons étudié six cas d'entreprises qui se sont internationalisées dès la création ou peu de temps après. Les caractéristiques de l'entrepreneur, ses compétences, son expérience ainsi que son réseau personnel sont importants pour la création de son entreprise à l'international et pour nouer les premiers contacts sur les marchés étrangers. La personnalité et le réseau de l'entrepreneur agissent en interaction; ils orientent tour à tour la création de l'entreprise et ses premiers pas sur les marchés étrangers.

\begin{abstract}
The study of International New Ventures (INVs) is becoming increasingly important in the entrepreneurship literature. Unlike traditional SMEs, INVs, also referred to as " born globals" (McKinsey \& Co., 1993) are defined as firms that from their inception or shortly after seek to derive a significant competitive advantage from resources use and sale of products in multiple countries (Oviatt and McDougall, 1994). Our research aims to understand how the entrepreneur's network and characteristics influence the internationalization of young firms. We studied six firms that internationalized at inception or shortly after and found that the entrepreneur's characteristics, skills, experience and personal network are important for creating an international venture and establishing initial contacts in foreign markets. Hence, the entrepreneurs' personality and network interact and guide the venture's internationalization process.
\end{abstract}

\title{
RESUMEN
}

El estudio de las Empresas Nuevamente Internacionales (ENI) está cobrando una importancia cada vez mayor en la literatura empresarial. A diferencia de las empresas tradicionales, estas ENI, también conocidas como «born globals» (McKinsey \& Co., 1993) o « international new ventures » (Oviatt y McDougall, 1994) se definen como empresas que, desde su inicio o poco después, tratan de obtener ventajas competitivas por el uso de recursos y venta de productos en varios países. Nuestra investigación tiene como objetivo entender cómo la red y las características del empresario intervienen en la internacionalización de las empresas jóvenes. Se estudiaron seis casos de empresas que se internacionalizaron al crearse o poco después. Las características del empresario, sus habilidades, experiencia y red de contactos personales son importantes para la creación de un negocio en el extranjero y establecer contactos iniciales en los mercados extranjeros. La personalidad y la red del empresario interactúan; guían sucesivamente la creación de la empresa y sus primeros pasos en los mercados extranjeros.

\section{ZUSAMMENFASSUNG}

Studien zu Born Globals (McKinsey \& Co., 1993) oder auch 'International New Ventures' (Oviatt und McDougall, 1994) nehmen in der Entrepreneurship-Literatur einen immer wichtigeren Platz ein. Im Gegenteil zu traditionell internationalisie- 
renden Unternehmen versuchen Born Globals direkt oder kurz nach der Gründung durch wirksames Einsetzen ihrer Ressourcen eine schnelle Internationalisierung zu erreichen um einen Konkurrenzvorteil zu erlangen. Vorliegende Studie untersucht, wie das Netzwerk sowie die Eigenschaften des Unternehmers auf die Internationalisierung wirken. Anhand von sechs Fallstudien wird die Wichtigkeit der Eigenschaften, der Kompetenzen, der Erfahrung und des persönlichen Netzwerkes des Unternehmers für die ersten internationalen Kontakte analysiert. Unternehmerpersönlichkeit und sein Netzwerk interagieren dabei und realisieren schrittweise die Gründung und Internationalisierung des Unternehmens.

\section{Introduction}

Oviatt et McDougall (1994) définissent les entreprises nouvellement internationales (ENI) comme toute entreprise qui, dès sa création ou peu de temps après, cherche à dériver un avantage concurrentiel de l'utilisation de ressources et de la vente de produits dans de multiples pays. Ces ENI ou «born globals» (McKinsey \& Co., 1993) occupent une place de plus en plus prépondérante dans la littérature de l'entrepreneuriat international (McDougall, Oviatt et Shrader, 2003). Il devient donc important de mener des recherches plus systématiques et plus orientées sur ces entreprises nouvelles et internationales (Rialp, Rialp et Knight, 2005). En effet, plus les entreprises s'internationalisent de manière précoce, plus le temps qu'elles prennent pour s'internationaliser est court. De ce fait, leur démarrage international diffère quelque peu par rapport à une internationalisation tardive (Baldegger et Schueffel, 2009). Parmi les auteurs fondateurs du champ de l'entrepreneuriat international, Oviatt et McDougall (1994) ainsi que Madsen et Servais (1997) mentionnent le réseau personnel de l'entrepreneur comme l'un des éléments clés favorisant l'internationalisation précoce ainsi que l'accès à des ressources à l'échelle internationale. Or, à ce jour, les études portant sur le réseau des ENI ne prennent que peu en compte le réseau personnel de l'entrepreneur ainsi que son expérience pour accéder à des ressources internationales et se développer internationalement pendant la période de gestation de l'entreprise (Coviello, 2006). Pour ces ENI, si un réseau commercial international prend forme dès ou peu de temps après la création, on peut supposer que ces liens émergent avant même l'internationalisation, pendant la période de gestation ou de démarrage de l'entreprise et que l'entrepreneur joue un rôle central. Par conséquent, si nous tenons compte, d'un côté, des caractéristiques de l'entrepreneur et, de l'autre, des réseaux formés pour créer et développer des échanges avec des marchés étrangers, nous serons en mesure de mieux comprendre l'internationalisation des jeunes entreprises.

L'objet de notre recherche exploratoire est donc d'étudier et de comprendre comment l'entrepreneur et son réseau favorisent l'internationalisation précoce de son entreprise. Nous centrons notre analyse sur les entreprises 
de la région Rhône-Alpes, deuxième région exportatrice française après l'Îlede-France. Dans un premier temps, afin de mieux comprendre la formation de réseaux à l'international chez les jeunes entreprises, nous intégrons la littérature sur le processus d'internationalisation et en entrepreneuriat international pour analyser la place de l'entrepreneur et de son réseau dans le développement international de son entreprise. Nous mettons en avant l'importance de la personne de l'entrepreneur, de son expérience ainsi que de son esprit international (Oviatt et McDougall, 1994, 1995) pour développer un réseau à l'international. Puis nous verrons comment le réseau de l'entrepreneur se confond avec celui de l'entreprise en formation pour développer des contacts et des relations commerciales sur les marchés étrangers. Dans un deuxième temps, nous présentons nos données relatives au démarrage international des entreprises en faisant ressortir les caractéristiques de l'entrepreneur et de son réseau. Nous centrons notre analyse sur les liens entre le démarrage, la personnalité de l'entrepreneur et les réseaux internationaux de ce dernier.

\section{La personne de l'entrepreneur et l'internationalisation de son entreprise: aspects conceptuels et méthodologiques}

C'est dans le domaine de l'entrepreneuriat international, qui constitue le point d'intersection de l'entrepreneuriat, de la gestion de la PME et de la gestion internationale, que s'inscrit cette partie qui a pour objectif d'attirer l'attention sur la relation entre la personnalité de l'entrepreneur, la constitution de son réseau et l'internationalisation rapide de son entreprise. $\mathrm{La}$ vision développée par l'entrepreneur en PME est par ailleurs susceptible d'influencer la mise en œuvre de l'internationalisation.

\subsection{L'influence de l'entrepreneur, de son expérience et de ses ressources dans l'internationalisation précoce de son entreprise}

Calof (1994) a trouvé que la petite taille n'entrave pas l'internationalisation de la PME, mais limite seulement le nombre de marchés qu'elle peut pénétrer. Nous pouvons en conclure avec Reid (1981) que quel que soit le type de facteurs favorisant l'apparition de stimuli à l'exportation, leurs reconnaissances et influences sur la décision d'exportation sont fonction des attitudes (motivations/personnalité), des compétences et de l'expérience 
managériales. Tan, Brewer et Liesch (2007) cherchent à «mesurer» cette implication de l'entrepreneur en élaborant un index ${ }^{1}$ et tentent de le relier au succès à l'export.

L'esprit international de l'entrepreneur (Oviatt et McDougall, 1994, $1995)$ apparaît alors comme le point de départ privilégié pour analyser ces ENI. C'est donc grâce à sa personnalité et à ses motivations tournées vers l'international que l'entrepreneur va se construire une vision mondiale, une réflexion (en amont) pour l'internationalisation. Cette vision est en partie appuyée par l'expérience professionnelle de l'entrepreneur; ensuite, cet entrepreneur va développer des compétences à l'international afin de se créer un réseau (Coviello, 2006); il va donc mobiliser ses ressources et accumuler progressivement une expérience professionnelle à la fois dans son secteur d'activité et à l'international (Oviatt et McDougall, 1994). Ces éléments, qui paraissent à priori intervenir de façon linéaire, s'enrichissent mutuellement; par exemple, l'expérience de l'entrepreneur peut impulser de nouvelles motivations, qui, à leur tour, lui ouvriront de nouvelles voies et lui fourniront donc de nouvelles ressources à explorer, etc.

L'esprit international de l'entrepreneur, son expérience et son réseau sont des éléments qui sont reliés. Nous proposons donc de les discuter simultanément en montrant leur imbrication au regard de l'internationalisation de la jeune entreprise.

\subsubsection{Attitudes, motivation pour l'international et esprit international}

C'est une tâche très ardue de mettre en relation les caractéristiques (attitude, motivations) de l'entrepreneur et l'internationalisation de l'entreprise. Ainsi, les résultats de Favre-Bonté et Giannelloni (2007) montrent l'absence d'effet direct des variables de personnalité (niveau de stimulation optimal, lieu de contrôle, aversion au risque) et du risque perçu lié à l'activité internationale sur la performance export des PME. En revanche, leur étude met clairement en évidence l'influence positive de l'implication de l'entrepreneur pour l'international en général. Ainsi, l'attitude de l'entrepreneur face à l'international est un facteur déterminant de son engagement sur les marchés étrangers. C'est précisément ce que montrent Autio, Sapienza et Almeida (2000): les entrepreneurs de ces ENI ont une attitude positive à l'égard de l'internationalisation et voient les marchés étrangers comme moins risqués que ceux des entreprises plus traditionnelles. Ils s'engagent donc plus facilement hors de leurs frontières. L'esprit international de l'entrepreneur chez ces ENI est donc un facteur important pour comprendre le développement international

1. IRI: Internationalisation Readiness Index. 
de l'entreprise (McDougall, Shane et Oviatt, 1994). Il permet de déceler des opportunités internationales et de développer le réseau international suffisant pour les explorer.

\subsubsection{Mobilisation des ressources de l'entrepreneur}

La littérature (McKinsey \& Co., 1993; Bell, McNaughton, Young et Crick, 2003) s'accorde pour dire que ces ENI possèdent souvent un avantage concurrentiel basé sur des ressources uniques qui leur permettent de proposer des produits et services à forte valeur ajoutée et/ou de se développer sur des segments de marché spécifiques. Ces entreprises possèdent donc des ressources et compétences inégalables (notoriété construite autour des compétences de l'entrepreneur, ressources-clés, etc.), liées, dans un premier temps au moins, à l'entrepreneur (Weerawardena, Mort, Liesch et Knight, 2007). Weerawardena et al. (2007) évoquent même l'existence de capacités dynamiques, construites par les entrepreneurs à profil international, qui servent de tremplin pour l'internationalisation de leur entreprise. La mobilisation de l'approche par les ressources (Barney, 1991) va nous permettre de mieux comprendre la formation de l'avantage concurrentiel et le démarrage précoce de ces entreprises à l'international. Ainsi, Ageron et Huault (2002) relèvent que le dirigeant d'entreprise va s'entourer d'acteurs (tels que les responsables fonctionnels) qui lui serviront de moteurs dans le processus de structuration de l'entreprise et dans son processus d'internationalisation. L'accumulation de connaissances autour de la personne de l'entrepreneur et la mise en place de compétences fonctionnelles spécifiques peuvent donc être vues dans ces entreprises comme une source d'avantages concurrentiels (Autio et al., 2000). L'entrepreneur par l'intermédiaire de son réseau mobilise les ressources nécessaires aux premières étapes de son internationalisation.

\subsubsection{Expérience internationale et professionnelle dans le secteur d'activité}

L'expérience antérieure de l'entrepreneur dans le secteur d'activité et son expérience personnelle à l'international (voyage, formation) vont lui permettre de concrétiser sa vision internationale à travers la constitution et l'activation de liens avec des acteurs étrangers et donc d'acquérir des compétences dans la gestion de ces liens. De façon générale, les connaissances et expériences antérieures réduisent la distance psychique ${ }^{2}$ (vs proximité culturelle)

2. Le concept de distance psychique peut être défini comme étant la somme des facteurs, tels que les différences de langue, de cultures, de pratiques commerciales, qui empêchent ou perturbent les flux d'information entre l'entreprise et le marché (cf. Johanson et Vahlne, 1990). 
ainsi que l'incertitude; elles jouent un rôle important dans l'accélération de la vitesse d'apprentissage (Chetty et Campbell-Hunt, 2004). En effet, l'expérience de l'entrepreneur peut être vue comme la somme des connaissances accumulées au fil des ans; elle représente le socle sur lequel l'entrepreneur va constituer les fondations et les orientations de sa nouvelle entreprise. Zucchella, Palamara et Denicolai (2007) montrent que l'expérience, en particulier l'expérience internationale accumulée au sein d'entreprises familiales ou de multinationales, est l'un des moteurs de l'internationalisation précoce. Par ailleurs, l'étude de Favre-Bonté et Giannelloni (2007) met clairement en évidence l'influence positive de l'expertise de l'entrepreneur en matière de procédures liées à l'international. McDougall et al. (1994) soulignent également l'importance de l'expérience de l'entrepreneur dans les affaires internationales, dans la détection d'opportunités internationales et la création d'entreprises immédiatement internationales. Ils voient les opportunités que d'autres ne voient pas parce que leurs connaissances (liées à leur parcours professionnel et international) sont uniques (Weerawardena et al., 2007). De plus, l'expérience internationale du créateur autorise à penser qu'il sera mieux à même de constituer un réseau international et de l'utiliser.

\subsection{Le rôle de l'entrepreneur dans la création du réseau à l'international}

Nous présentons ci-après quelques-unes des principales approches liant l'internationalisation et le concept de réseau ${ }^{3}$. Nous centrons notre analyse sur la place qu'occupe le concept de réseau dans la théorie des nouvelles entreprises internationales d'Oviatt et McDougall (1994) et du point de vue du champ de l'entrepreneuriat. Deux courants de recherche à l'intérieur du champ de l'entrepreneuriat s'intéressent à la notion de réseau: les réseaux interorganisationnels ou les réseaux étendus, d'une part, et les réseaux personnels, d'autre part. Dans le cadre de notre travail, nous nous concentrons, dans un premier temps, sur le réseau personnel de l'entrepreneur tel qu'il est observé dans le processus de création d'entreprise (1.2.1). Puis, nous regardons comment le réseau personnel de l'entrepreneur se confond avec celui de l'entreprise créée à l'international (1.2.2).

3. Nous ne parlerons pas de l'approche «network» de l'internationalisation qui met l'accent sur les relations «réseau» au niveau d'une industrie en général. Le lecteur pourra se référer aux travaux de Johanson et Mattson (1988) pour de plus amples discussions sur le modèle réseau de l'internationalisation, qui est considéré comme une extension du modèle d'internationalisation d'Uppsala (modèle U). 


\subsubsection{L'impact des réseaux personnels sur la création d'entreprise}

Le processus de création de l'entreprise correspond à une période de temps où les besoins en ressources sont importants afin de réussir le démarrage. Le réseau est donc un moyen de pallier le manque de ressources et d'accéder à des éléments d'actif requis pour l'entreprise (Oviatt et McDougall, 1994). Nous centrons notre analyse sur le réseau personnel de l'entrepreneur tel qu'il est appréhendé dans la littérature en entrepreneuriat international (McDougall et al., 1994).

La notion de réseau ${ }^{4}$ est très employée par les chercheurs étudiant le processus entrepreneurial (Dodd, 1997); le contexte environnemental dans lequel l'entrepreneur évolue peut être appréhendé à travers les réseaux existants à plusieurs niveaux (relations entre les individus, les groupes et les organisations; Dubini et Aldrich, 1991) ou bien encore par les liens sociaux ou économiques (Johannisson, 1987).

Le réseau personnel de l'entrepreneur est important en fin de période de gestation de l'entreprise, mais aussi à son démarrage, en particulier à l'international (Ojala, 2009). La disponibilité et le développement des réseaux personnels expliquent pourquoi certaines personnes créent des entreprises et d'autres pas (Aldrich et Zimmer, 1986; Johannisson, 1987). Il est aussi souvent admis que les informations nécessaires pour démarrer une entreprise sont souvent transmises à l'entreprise à travers un réseau d'amis et de connaissances plus ou moins proches (Johannisson, 1987). Pour d'autres (Ostgaard et Birley, 1994), le réseau personnel du propriétaire-dirigeant est la plus importante ressource sur laquelle il se repose. Les bénéfices de la création et de l'entretien d'un réseau sont divers et variés, même s'il n'existe pas de consensus en la matière; on retrouve notamment le partage d'informations, l'extension du nombre des contacts, la présentation à des partenaires, l'avancée d'idées (O’Donnell, Gilmore, Cummins et Carson, 2001).

Le terme «réseautage ${ }^{5}$ » décrit un comportement entrepreneurial; il correspond à certains types de relations à l'intérieur des réseaux personnels. Il s'agit d'un réseau construit sur des liens ${ }^{6}$ forts sur lesquels l'entrepreneur peut compter (Dubini et Aldrich, 1991) et qui s'inscrivent dans une relation à long terme. Les entrepreneurs sont en mesure d'augmenter leur champ d'action à travers leur réseau personnel et d'accéder ainsi, à un coût limité,

4. Le réseau consiste en une série de liens directs et indirects d'un acteur à un ensemble d'autres acteurs.

5. Le terme réseautage correspond au mot anglais «networking».

6. Par contraste, les liens faibles sont superficiels ou occasionnels, et les personnes n'ont pas tendance à s'investir sur le plan émotionnel à leur égard. 
à des ressources autrement indisponibles. Tous les entrepreneurs, durant la période de création, s'appuient sur leurs réseaux personnels existants et en établissent de nouveaux au cours du processus afin d'obtenir des ressources et des connaissances pour leurs organisations (Weerawardena et al., 2007). Ces réseaux permettent, entre autres, à l'organisation émergente de s'orienter à l'international dès sa formation.

\subsubsection{La transformation des réseaux personnels en réseaux interorganisationnels}

Pour Johannisson, Alexanderson et Senneseth (1994), en ce qui concerne la recherche en entrepreneuriat, la séparation entre les échanges interpersonnels et interorganisationnels est, dans une grande mesure, dépassée. L'entrepreneur incarne son entreprise; il doit personnellement intégrer les diverses dimensions sociales et économiques des liens du réseau. Ainsi, il n'est pas rare que les recherches en entrepreneuriat sur le réseau personnel de l'entrepreneur incluent à la fois des liens formels et informels entre les individus, et d'autres, entre les individus et les organisations au sein de leur contexte environnemental. La transformation des réseaux personnels en des réseaux étendus ou interorganisationnels est particulièrement cruciale pour les très petites entreprises ou les entreprises en création.

L'approche de l'entrepreneuriat par les réseaux sociaux met en avant des entrepreneurs «enracinés» dans un contexte social. Ces derniers sont contraints, inhibés, dirigés ou aidés par leur position dans les réseaux sociaux (Aldrich et Zimmer, 1986; Granovetter, 1985). Le réseautage n'est pas simplement une activité individuelle (de l'entrepreneur), mais émane aussi des membres de l'organisation. Pour Dubini et Aldrich (1991), l'entrepreneur n'est pas considéré comme la seule personne responsable pour saisir les opportunités, mais l'entreprise tout entière développe une conscience de son environnement qui scrute son territoire, même au-delà des limites nationales. Au démarrage de l'entreprise, il n'y a donc pas lieu de distinguer les deux réseaux (personnel et interorganisationnel) car, chez les ENI, ils se confondent en s'enrichissant mutuellement, notamment pour les activités à l'international. L'entrepreneur active et développe un réseau international qu'il met au service de l'organisation émergente.

\subsection{Les liens entre l'entrepreneur, les réseaux et le développement international de la jeune entreprise}

Le réseau est donc un moyen d'accéder à des ressources à l'international sans les posséder. McDougall et al. (1994) expliquent que les réseaux de l'entrepreneur aident les créateurs à identifier des opportunités d'affaires à 
l'international et que ces réseaux ont plus d'influence sur le choix des pays pénétrés que la distance psychique. Les opportunités internationales et la trajectoire suivie par l'entreprise dépendent avant tout des liens existants et en cours de création avec, notamment, les clients et les fournisseurs de l'entreprise. Oviatt et McDougall (1995) soutiennent que les réseaux d'affaires à l'international développés constituent l'une des sept plus importantes caractéristiques pour expliquer la réussite de ces entreprises dites «global start-ups». Servais et Rasmussen (2000) trouvent que les réseaux s'avèrent critiques pour l'internationalisation de la majorité des entreprises danoises de leur étude. Ainsi, les réseaux de l'entrepreneur et de son équipe façonnent et dirigent l'internationalisation de l'entreprise en formation, ce qui explique pourquoi, vue de l'extérieur, l'internationalisation de l'entreprise semble due au hasard ou irrationnelle (Coviello et Munro, 1997). En somme, l'étendue et le développement des activités internationales de l'entreprise sont reliés aux opportunités qui émergent du réseau de relations de l'entrepreneur et de l'entreprise.

Par ailleurs, l'importance des relations interpersonnelles (qui permettent l'accumulation de connaissances, la création et le développement de nouvelles activités) a été mise en avant pour expliquer le processus d'internationalisation des entreprises (Coviello et McAuley, 1999). Les relations interpersonnelles sont la base pour la création de nouveaux réseaux interorganisationnels (Harris et Wheeler, 2005). De cette manière, les relations personnelles et directes autorisent l'accès à de nouveaux marchés et de nouvelles ressources (Ellis, 2000) et apportent aussi des connaissances pour les marchés internationaux sur lesquels l'entrepreneur souhaite développer son activité (Harris et Wheeler, 2005). Ces relations proviennent à la fois du contexte social et des activités d'affaires de l'entrepreneur (Ellis et Pecotich, 2001).

Les réseaux personnels de l'entrepreneur et les réseaux étendus de l'entreprise se relient et se confondent pour expliquer le démarrage et le développement international des jeunes entreprises. Une fois l'entreprise créée, le réseau de contacts internationaux (étendu ou interorganisationnel) se développe, les relations s'institutionnalisent et, à terme, le réseau étendu de l'entreprise se disjoint du réseau de l'entrepreneur ou de ses collaborateurs. Ces relations réseau, à la fois au niveau de l'individu et de l'entreprise, façonnent et orientent les premières étapes de l'internationalisation de l'entreprise. Elles peuvent même évoluer et se transformer au cours du temps avec le développement de l'entreprise, grâce aux activités de réseautage de l'entrepreneur. Une véritable dynamique se met alors en place. 
FIGURE 1

Le démarrage international des jeunes entreprises

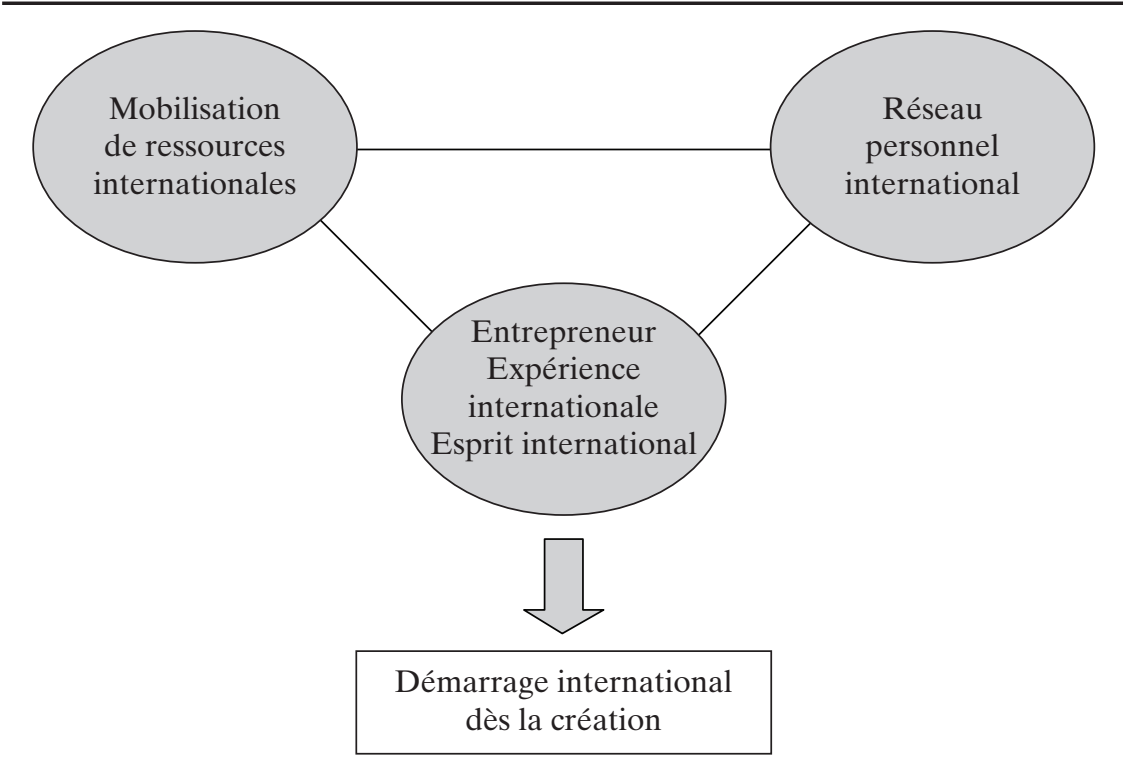

Nous venons de voir que l'entrepreneur et son réseau personnel influencent l'internationalisation, avant même que l'entreprise ne soit créée (figure 1). Aussi, est-il intéressant, pour étudier le phénomène des nouvelles entreprises internationales, de tenir compte du contexte de leur création. Cette démarche a pour objectif de montrer que l'internationalisation remonte bien avant la constitution de l'entité. Nous présentons ci-dessous la méthodologie utilisée pour mieux comprendre le rôle des réseaux lors du démarrage international des jeunes entreprises.

\subsection{Méthodologie: six études de cas portant sur le réseau international de l'entrepreneur}

Après avoir présenté notre méthode de recherche, nous exposons les caractéristiques des ENI qui nous ont servi de terrain d'étude.

\subsubsection{Méthodologie retenue et choix de l'échantillon}

La question de recherche guidant notre étude est caractérisée à la fois par la description et l'interprétation. Nous avons opté pour une méthodologie basée sur l'étude de cas; celle-ci est particulièrement pertinente lorsque l'objectif 
de recherche est d'explorer, de comprendre ou de générer des idées sur le pourquoi et le comment. Pour notre travail, nous utilisons les méthodologies décrites par Yin (1989) et Miles et Huberman (1994). Comme pour les échantillons destinés à des traitements quantitatifs, la taille d'un échantillon pour un traitement qualitatif dépend de l'objectif poursuivi (Royer et Zarlowski, 1999). Nous avons choisi de traiter plusieurs cas. La taille de l'échantillon est définie selon deux principes qui s'appliquent autant pour des cas que pour des répondants: la saturation et la réplication. Comme recommandé par Eisenhardt (1989), les sites ont été sélectionnés pour des raisons théoriques. La représentativité théorique s'obtient en choisissant des cas représentatifs des contrastes et des différentes modalités des concepts mobilisés. Lorsque les acquis théoriques existent, l'observation de multiples situations permet de s'intéresser aux régularités et aux différences pour discuter de la validité.

Nous avons repéré 25 entreprises de la région Rhône-Alpes, qui ont développé un produit, qui se sont internationalisées avant l'âge de six ans ${ }^{7}$ et qui ont été créées ex nihilo par une personne physique après 1996. Sur les 25 cas, nous en avons retenu six. Pour la sélection, nous avons tenu compte de la variété des secteurs d'activité, de la répartition des entreprises sur la région Rhône-Alpes ainsi que le volume des ventes à l'international. Les entreprises sélectionnées sont des entreprises orientées produits, ayant une composante technologique importante; elles sont réparties sur toute la région Rhône-Alpes et représentent différents secteurs d'activité. Tous les entrepreneurs contactés ont accepté de nous rencontrer et d'échanger des données relatives à l'entreprise et à son secteur.

Nous focalisons notre analyse sur les activités commerciales des entreprises. Nous avons sélectionné nos cas afin d'observer toutes les situations possibles et d'appréhender le développement international des jeunes entreprises dans sa globalité. Nous nous concentrons seulement sur les premières étapes de l'internationalisation. Les entreprises sélectionnées, de tailles différentes, appartiennent à des secteurs variés (industrie classique, logiciel, haute technologie) et sont toutes axées sur le développement d'une technologie. Par ailleurs, elles cherchent à dériver un avantage concurrentiel significatif de l'utilisation de ressources et de la vente de produits dans de multiples pays (Oviatt et McDougall, 1994).

7. Oviatt et McDougall (1997) soutiennent que l'âge de six ans est l'âge limite en dessous duquel une entreprise peut être considérée comme ayant eu une internationalisation rapide et précoce. 


\subsubsection{Collecte et traitement des données}

Nous avons réalisé une série d'entretiens semi-directifs sur chaque site en utilisant les procédures mises en avant par Yin (1989) et Eisenhardt (1989) ainsi qu'un guide d'entretien thématique. Deux entretiens par site ont été effectués avec l'entrepreneur créateur. Nous avons aussi rencontré le responsable export quand celui-ci n'était pas l'entrepreneur lui-même. Différentes recherches d'informations ont été effectuées pour compléter et vérifier les déclarations en examinant des données secondaires (documents, recherche sectorielle, outils de promotion).

Les données recueillies ont été organisées selon les techniques préconisées par Miles et Huberman (1994) en effectuant un travail de condensation des données, de présentation, d'élaboration et de vérifications des conclusions à chaque étape d'analyse. Notre codage est essentiellement un «codage thématique» à priori défini par Miles et Huberman (1994). Il classe les unités d'analyse dans des catégories qui regroupent, entre autres, les difficultés, les conséquences, les moyens et les actions du phénomène. Il est à noter que nous n'utilisons pas de codes chronologiques afin de ne pas surcharger le codage et l'analyse en multipliant les catégories. L'ordre des événements est établi par grande thématique ou à l'aide de codes explicatifs qui permettent, pour certains, de retracer l'enchaînement temporel des données. Dans chaque thématique, nous avons mélangé les codes descriptifs et explicatifs. Au total, quatre thématiques ont été relevées : la création d'entreprise, l'organisation, le processus d'internationalisation et le réseau.

Elles ont servi de base pour le guide d'entretien et pour la création des codes. Par ailleurs, nous utilisons aussi, pour la définition de nos codes, les analyses issues de notre premier travail exploratoire des entretiens avec un certain nombre d'experts rencontrés (agences export, structures d'accompagnement...). Deux chercheurs ont établi chacun leur codage et ont échangé leur point de vue sur chacune des thématiques. Ils ont confronté leurs différences de codes une fois le codage terminé et ont échangé sur la création de nouveaux codes plus précis, notamment, sur la thématique «réseau» ainsi que sur les caractéristiques du réseau. Par ailleurs, ils ont confronté leur codage avec l'avis de deux conseillers «export» de deux chambres de commerce et d'industries situées dans la région Rhône-Alpes. Une fois codées, les données ont été classées par site et par type de codes en tenant compte de la chronologie des événements. Pour la comparaison, une matrice multisite chronologique a été réalisée autour du concept de réseau. La multiplication et les comparaisons de sites font que notre méthode qualitative s'apparente à « l'étude de cas collective» (Stake, 1994) qui offre au chercheur des cas multiples dans l'optique de s'informer sur un phénomène. Pour les 
besoins de la présente étude, nous avons sélectionné six cas. Nous présentons ci-après quelques-unes de nos principales analyses qui ont trait au démarrage international, aux caractéristiques de l'entrepreneur et à son réseau.

\section{Les spécificités de l'internationalisation précoce de nos entreprises: résultats et implications}

Nous présentons dans un premier temps quelques données relatives aux entreprises et à leur démarrage international afin de rendre compte des spécificités de leur internationalisation précoce. Puis, nous abordons l'influence des caractéristiques de l'entrepreneur (expériences, esprit international, parcours) sur les premiers pas à l'international de l'entreprise. Cette étape nous amène ensuite à étudier quelles sont les principales caractéristiques du réseau international pour les six cas étudiés à partir des critères d'analyse du réseau de Coviello (2006). Notre objectif est d'abord d'effectuer une analyse intersite afin de repérer les similitudes et les différences entre nos cas; il s'agit d'observer si les entrepreneurs et leur entreprise ont le même comportement à l'international lors du démarrage de l'activité. Enfin, nous terminons par les implications pratiques et théoriques de notre étude.

\subsection{Un démarrage des ventes à l'international proactif et rapide}

Nous résumons quelques données relatives au démarrage international des entreprises étudiées dans le tableau ci-contre.

Tous les entrepreneurs ont décidé d'internationaliser leur entreprise avant sa création et ont entrepris des démarches «export» avant qu'une entité ne soit créée. Dès la première année d'internationalisation, quatre entreprises ont un chiffre d'affaires à l'international supérieur à $50 \%$. En 2002, toutes les entreprises ont un CA à l'international supérieur à $70 \%$.

Les entrepreneurs décident de manière volontaire et active d'orienter la création et/ou le développement de leur entreprise à l'international; ils s'impliquent directement dans le processus. Il n'y a, dans l'ensemble, pas de période d'attente ou d'hésitation. Le démarrage international est très rapide; il est soit accéléré (TI), soit quasi instantané (RA, DG, DN, TR, DS). Pour toutes les entreprises, les entrepreneurs engagent dès le départ des ressources importantes (financières, humaines, en temps) pour démarrer et développer l'internationalisation. 


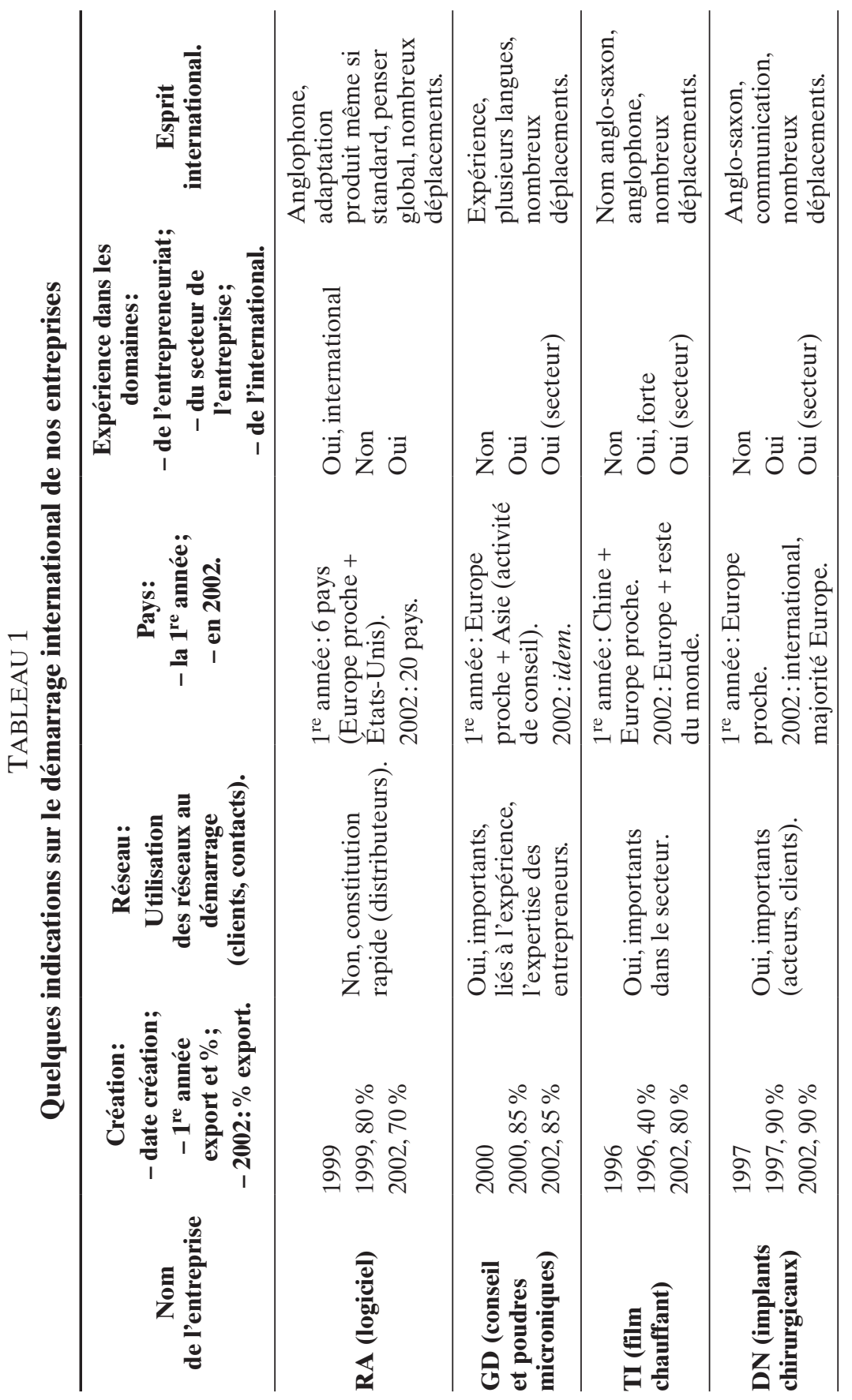




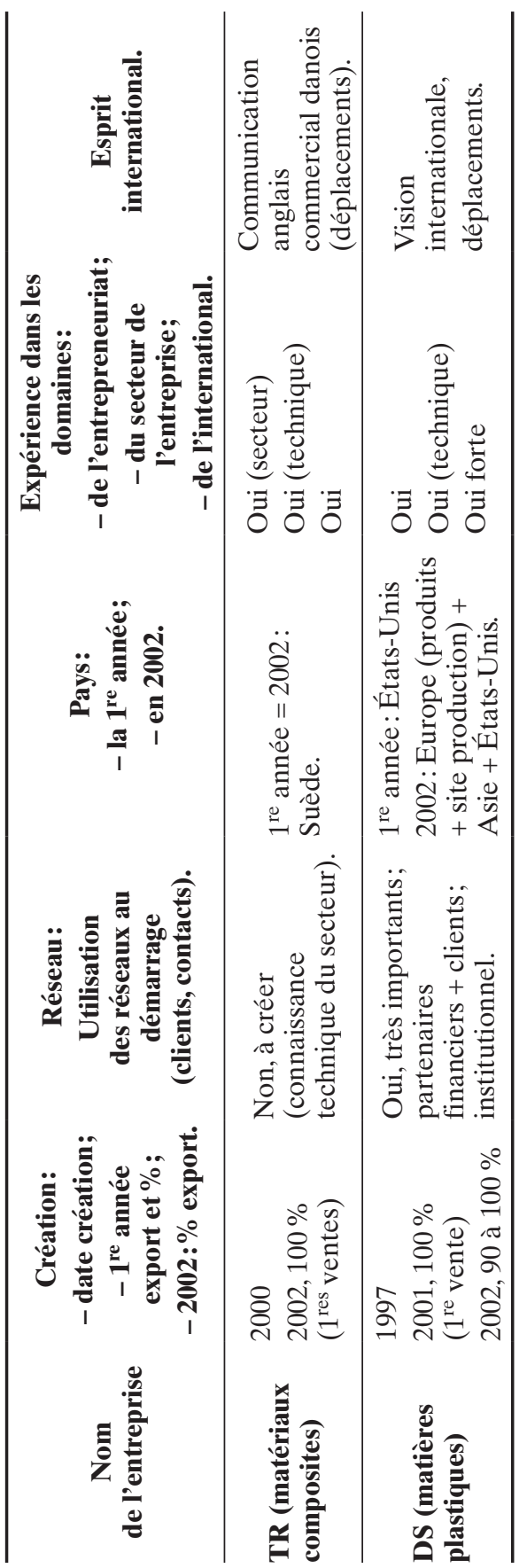

Revue internationale P.M.E., vol. 24, nº 2, 2011 
Le principal mode de développement utilisé est l'exportation directe et les distributeurs ou agents. Certaines entreprises, compte tenu de la nature de leurs produits ou de leur technologie, établissent des accords de licence (DS, TR). Dans un cas, il y a création d'une filiale (DN) qui correspond plus à la saisie d'opportunités qu'à une véritable logique d'implantation. La première année, toutes les entreprises ont une présence en Europe (sauf DS). Cependant, pour cinq entreprises, la commercialisation sur le marché européen se réalise en même temps que sur d'autres zones géographiques beaucoup plus éloignées. Ainsi, la proximité géographique et culturelle de l'Europe intervient, mais ce n'est pas le seul critère de sélection: l'importance du marché, son homogénéité, les contacts établis ou encore les ressources financières disponibles sont également importants. Ainsi, dès 2002, on constate une forte diversité géographique. Dans l'ensemble, le démarrage international s'effectue dès la création ou peu de temps après, de manière rapide et proactive. L'activité internationale devient alors très vite prépondérante pour toutes les entreprises, contrairement au processus d'internationalisation décrit par Johanson et Vahlne (1990).

\subsection{Des entrepreneurs aux caractéristiques très marquées (cf. annexe l)}

\subsubsection{L'expérience des entrepreneurs au service de leur entreprise}

Toutes les entreprises ont au moins un créateur qui possède une expérience professionnelle dans le même secteur d'activité que l'entreprise créée (cinq cas sur six, $c f$. le tableau 1). Tous les entrepreneurs ont déjà une expérience internationale. Les créateurs ont soit une forte expérience internationale dans le même secteur d'activité de l'entreprise, soit une forte expérience dans les affaires internationales (DS, TR). Les entrepreneurs utilisent leurs expériences internationales pour continuer à développer des contacts internationaux avant la création, principalement sur le plan commercial (mais pas seulement), ainsi que postcréation. Forts de leurs expériences, ils peuvent développer leurs réseaux sur différents marchés étrangers et orienter les premiers pas de leur entreprise à l'international.

\subsubsection{La création d'un esprit international}

Dans tous les cas, l'entrepreneur a cherché à développer un esprit international dès l'idée de création. Cet esprit revêt différentes caractéristiques selon les cas et c'est lui qui oriente le projet de création. Ce qui correspond à la notion de «global vision» d'Oviatt et McDougall (1995). Les verbatims 
ci-dessous illustrent cet esprit international. Pour les créateurs de GD, l'international fait partie de leur projet de création et est apparu naturellement: «on a travaillé dans un grand groupe, donc les relations internationales, pour nous, c'était naturel, on avait déjà l'expérience». Les créateurs de l'entreprise RA ne se sont pas posés de question pour aller à l'international: «la démarche export ne nous a pas tellement effrayés. On est parti un peu la fleur au fusil dans le domaine, mais on n'a pas eu de difficultés particulières... Il faut vraiment penser global».

Dans tous les cas, l'entreprise a réfléchi à un nom international, c'est-à-dire un nom latin ou à consonance anglo-saxonne. L'esprit international se retrouve également dans le développement des produits qui est résolument orienté vers l'international. L'entreprise RA, par exemple, décide dès la phase de conception des produits d'avoir un output le plus standard possible pour l'adapter à tous les marchés et aux besoins des entreprises. De plus, la plupart des produits des entreprises incorporent des aspects technologiques qui, par essence, ne demandent pas beaucoup d'adaptation sur le plan local.

Pour cinq cas, les créateurs s'occupent personnellement des démarches internationales en mettant à profit leur compétence internationale: ils ne focalisent pas leur étude de marché uniquement sur la France et entreprennent même, avant la création de l'entreprise, des voyages à l'étranger. Ainsi, les créateurs de GD n'hésitent pas à tout risquer pour l'international: «vous savez, la première année où l'on s'est lancé, on a fait le plus grand salon [du secteur] aux États-Unis, on avait un budget de 100000 francs alors que notre chiffre d'affaires de l'année a été de 400000 francs... Il faut être capable de prendre des risques à un moment donné... il faut avoir l'esprit international pour le faire». Trois entreprises (RA, DN et TR) introduisent une communication en anglais (rapports, discussions, publicité, site Internet...). De manière anecdotique, l'entreprise RA a d'abord rédigé en anglais puis a fait traduire ses contrats en français.

$\mathrm{Au}$ total, l'ensemble de ces démarches prouve que l'international est intégré en amont, dès le projet de création de l'entreprise. Cette orientation internationale passe par la création d'un esprit international qui irrigue l'ensemble de l'organisation en émergence. Le réseau personnel de l'entrepreneur sert l'organisation tout entière dans la structure et l'orientation de ses démarches commerciales. La partie suivante va s'attacher à montrer comment les entrepreneurs créent et développent des contacts à l'international pour le démarrage de l'entreprise. 


\subsection{Une forte mobilisation du réseau pour le démarrage international}

\subsubsection{L'importance du réseau international de l'entrepreneur}

La capacité de l'entrepreneur à créer un réseau d'informations et de contacts se révèle primordiale (aptitude au réseautage) avant même la création de leur entreprise. Le réseau à l'international des entrepreneurs intervient à plusieurs niveaux. Par exemple, il peut être utilisé pour acquérir de l'information sur les marchés étrangers. Il sert parfois d'intermédiaire pour accéder à de futurs clients ou prospects. Il joue enfin un rôle majeur pour les modes de développement utilisés sur les différents marchés étrangers.

À titre d'exemple, les créateurs de DN (anciens responsables export), de GD ou de TI connaissent personnellement tous les principaux acteurs du marché. Les relations sont établies dans un premier temps de cette manière, ce qui leur permet d'éviter une prospection directe et d'avoir un meilleur accueil pour leurs produits. De façon similaire, le créateur de DS dispose d'un important réseau personnel et d'une forte aptitude au réseautage. Il réalise ses premières ventes internationales par l'intermédiaire d'un partenaire financier. Il envoie l'un de ses amis et partenaire s'occuper de la prospection sur le marché anglais et utilise son premier contact asiatique pour être présent en Russie et dans d'autres pays de l'ex-URSS.

Les créateurs de TI ont une très bonne connaissance des clients et des fournisseurs à l'étranger. Ils utilisent directement leurs contacts pour conclure leurs premières ventes à l'international. Ces contacts orientent ensuite l'entreprise. «On demande à un ami, s'il connaît des gens dans tel ou tel pays pour vendre; beaucoup de contacts arrivent comme ça. C'est beaucoup plus efficace que d'aller prospecter directement.» À l'inverse, le créateur de TR, malgré son expérience internationale, n'avait pas de contact particulier. Le cadre danois, embauché dès le départ en amont du projet, a mis à contribution ses réseaux et contacts internationaux dans des domaines d'activités parallèles à l'entreprise dans les pays du nord de l'Europe. La première vente a été réalisée en Suède et d'autres contacts commerciaux ont suivi dans cette région.

Ces contacts internationaux servent aussi à la création d'un réseau de distributeurs ou d'agents. L'animation de ce réseau permet à l'entreprise d'accéder aux marchés étrangers à moindres coûts et d'obtenir des informations. Ainsi, le réseau personnel des entrepreneurs ou des membres de l'équipe ainsi que leurs aptitudes pour l'activité de réseautage (constitution de réseaux commerciaux, financiers, d'informations, de fournisseurs) sont mis à contribution pour démarrer et développer les ventes à l'international. 


\subsubsection{Les caractéristiques du réseau international des ENI étudiées}

Il nous semble intéressant désormais de commenter les résultats relatifs aux caractéristiques structurelles et interactionnelles du réseau de nos ENI telles qu'elles ont été présentées par Coviello (2006). Nos données montrent que l'entrepreneur développe à l'international des relations de nature économique, mais qui peuvent avoir, dans certains cas, au fur et à mesure que la relation se renforce, un lien plus «social». Dans tous les cas, l'entrepreneur développe une capacité à créer des liens à l'international pour commercialiser ses produits. Notons également que ces liens avec l'international peuvent être créés par des entreprises étrangères qui contactent directement l'entreprise française pour établir une relation commerciale (cas de TI et DS), notamment par le biais d'Internet ou du repérage de l'entreprise sur un salon professionnel.

Tout l'enjeu pour ces entreprises est de réussir malgré leurs faibles ressources à augmenter la taille de leur réseau à l'international tout en densifiant les liens existants. Les contacts passés et nouveaux de l'entrepreneur lui permettent d'établir sur un nombre croissant de marchés son réseau commercial, qui est constitué principalement (en plus de l'exportation directe) d'agents et de distributeurs sur chacun des marchés ciblés.

Dans tous les cas, l'internationalisation est influencée par le réseau des relations commerciales internationales de l'entreprise et la nature des liens établis (liens forts ou faibles, densité, taille du réseau). Le réseau est un moyen d'accéder plus rapidement à certains marchés étrangers et d'entrer directement en contact avec les futurs clients de l'entreprise. Ce réseau permet en outre à l'entrepreneur de déceler de nouvelles opportunités commerciales et d'accéder à de nouvelles ressources. Il oriente aussi le choix des pays ciblés ainsi que l'intensité des relations avec les partenaires contactés dans chaque pays. Une fois les relations commerciales établies, il revient à l'entrepreneur d'entretenir son réseau de relations commerciales. Nous terminons cette communication en discutant des implications de l'existence de liens entre la personnalité de l'entrepreneur, son réseau et le démarrage international de son entreprise.

\subsection{Discussions et implications théoriques}

Nos résultats vont dans le sens de McDougall et al. (1994) qui expliquent que les réseaux aident les créateurs d' «international new ventures» ou de «born globals» à repérer les opportunités internationales et que ces réseaux semblent parfois avoir plus d'influence sur les choix du pays que la distance «psychique» (cas des entreprises DN, TR et DS par exemple). Les réseaux 
facilitent l'internationalisation rapide de ces nouvelles entreprises (Oviatt et McDougall, 1994; Coviello et Munro, 1997). Pour Shane (2000), le développement d'une nouvelle organisation peut être marqué par les liens et les connaissances engendrées par sa création (période de gestation de l'entreprise). Aussi, selon Coviello (2006), est-il nécessaire de comprendre les réseaux des «international new ventures » pendant les périodes d'internationalisation, de préinternationalisation, mais aussi de précréation (période de gestation). Bien que l'internationalisation ne survienne que dans les stades de développement et de croissance de l'entreprise, dans certains cas, elle est le résultat d'un lien établi beaucoup plus tôt dans le cycle de vie de l'entreprise (comme c'est le cas pour les entreprises DN, GD et TI).

Nos résultats appuient ceux issus des études de cas de Coviello (2006), car ils montrent que pour comprendre les transactions commerciales établies sur les marchés étrangers, il est nécessaire d'élargir la période de temps pris en compte en intégrant les phases de préinternationalisation et de gestation de l'entreprise. Avant même qu'une activité commerciale ne soit initiée, des liens sont créés et développés à l'international. L'origine des liens dans certains cas est liée à l'expérience de l'entrepreneur dans le secteur et/ou dans les affaires internationales avant la création de l'entreprise.

De plus, ces liens internationaux ne sont possibles que s'il existe une réelle volonté de l'entrepreneur d'orienter dès le début son activité entrepreneuriale vers l'international. Ce faisant, l'entrepreneur mobilise ses ressources pour créer des liens sur les différents marchés étrangers et développe ainsi un esprit international (Oviatt et McDougall, 1994; Andersson et Evangelista, 2006) propice à la création d'un réseau commercial international. Pour Oviatt et McDougall (1994) et Madsen et Servais (1997), l'entrepreneur dispose d'une vision internationale dès la création de l'entreprise. Nos résultats confirment aussi ceux de Coviello et Munro (1997) et Sharma et Blomstermo (2003) qui soulignent l'importance des toutes premières relations internationales pour le développement des nouvelles entreprises. Nos résultats montrent également que les liens créés sont susceptibles d'être de nature différente: ce sont soit des liens «sociaux» comme la famille, des amis ou des collègues (Ellis et Pecotich, 2001; Harris et Wheeler, 2005), soit des liens plus économiques liés à une relation d'affaires (Coviello, 2006). Ces liens, quelle que soit leur nature, permettent de créer et de développer des contacts et des relations internationales durables. Ils permettent d'optimiser le développement international de l'entreprise en formation en limitant les ressources mobilisées. De plus, les réseaux de nature économique ou sociale se mélangent ou se confondent parfois (cas des entreprises DS et GD). Par ailleurs, les réseaux 
personnels liés à l'expérience passée de l'entrepreneur se révèlent dans certains cas déterminants pour rendre compte de l'internationalisation de nouvelles entreprises.

\section{Conclusion, limites et perspectives}

L'entrepreneur et son réseau favorisent le démarrage international des jeunes entreprises étudiées. Ils orientent les premiers pas de ces entreprises sur les marchés étrangers. Cependant, il ne faut pas en déduire que l'entrepreneur, parce qu'il joue un rôle central, agit tout seul. En effet, il est souvent entouré d'une équipe, informelle la plupart de temps, de collaborateurs, d'amis, de membres de la famille, mais il sait également se constituer un réseau d'acteurs qui va lui apporter les compétences requises pour mener son projet international à terme.

Les résultats de notre recherche (avant tout exploratoire et basée sur six études de cas) soulignent l'importance de l'entrepreneur et de son réseau non seulement pour le démarrage international des nouvelles entreprises, mais également pendant la phase qui précède la création.

L'étude de nos différents cas montre également que le réseau international revêt différentes formes et qu'il peut être de diverses natures; il se densifie, se développe et change de forme au cours du temps. Aussi est-il difficile de dégager un modèle de démarrage international en tenant compte des interactions liées au réseau de l'entrepreneur. Cependant, l'analyse des six cas montre que le réseau international de l'entrepreneur se confond avec celui de l'entreprise à sa création. Par ailleurs, il apparaît nécessaire d'élargir la dimension temporelle de l'internationalisation en prenant en compte l'expérience passée de l'entrepreneur, son esprit orienté «international» et le début de ses activités entrepreneuriales qui façonnent les futures relations internationales.

Nos résultats sont à modérer en fonction des limites inhérentes à notre recherche. Tout d'abord, nous nous situons dans une démarche exploratoire basée sur six études de cas. Nous étudions des entreprises de différentes industries et nous ne contrôlons pas la variable sectorielle. Notre méthodologie est basée essentiellement sur l'exactitude de données fournies par les répondants eux-mêmes; l'analyse et les caractérisations des liens sont basées sur leur discours. Au niveau de la description de la nature des liens du réseau, nous sommes restés à un niveau d'analyse général sans tenir compte d'éléments plus détaillés susceptibles d'être mesurés comme l'importance, le niveau de confiance ou encore la fréquence de ceux-ci. 
Nous n'intégrons pas dans notre analyse la performance de l'entreprise. Ces différentes limites ouvrent la voie à de nouvelles recherches sur les différentes dimensions du réseau international des nouvelles entreprises. Il est possible de multiplier les études de cas en prenant en compte les différents contextes des entreprises. Des approches plus longitudinales intégrant une dimension temporelle élargie de l'internationalisation seraient utiles pour la compréhension du phénomène.

Actuellement, c'est dans cette voie que nous travaillons puisque nous étudions de nouveau les différents cas présentés afin de visionner les trajectoires parcourues en cinq ans.

Enfin, d'un point de vue théorique, l'intégration de la littérature sur les réseaux en entrepreneuriat et en entrepreneuriat international en adoptant un espace temporel élargi pourrait être utile à de futures recherches pour comprendre l'influence du réseau de l'entrepreneur sur le démarrage international des jeunes entreprises.

L'analyse du tableau en annexe nous montre que tous les entrepreneurs démarrent leurs projets en équipe. Ils ont tous une formation technique sauf pour une personne. Ils sont majoritairement ingénieurs. Les différents cas montrent qu'ils créent directement dans le même secteur où ils ont acquis de l'expérience au cours des années, dans le même domaine d'activité ou dans un segment de marché proche. Au moins un des entrepreneurs a une expérience commerciale du marché à l'international importante (quatre cas) ou une expérience professionnelle internationale importante dans un autre secteur (DS) ou bien encore l'expérience d'un projet de création à l'international avec l'Asie (RA). Quand ils n'ont pas d'expérience commerciale dans le secteur, les entrepreneurs utilisent leur expérience technique et leurs connaissances du secteur à l'international ainsi que leur expérience internationale personnelle ou dans d'autres secteurs pour effectuer leur démarrage commercial à l'international. Cette expérience internationale se renforce avec la création et le démarrage à l'international. Pour les entrepreneurs ne disposant pas de contacts commerciaux lors du processus de création, ils capitalisent sur leur expérience professionnelle à l'international de l'un des créateurs pour créer rapidement des contacts commerciaux à l'étranger. L'accent est mis sur l'utilisation de cette expérience au profit de l'équipe qui se structure ainsi que de l'entreprise. 


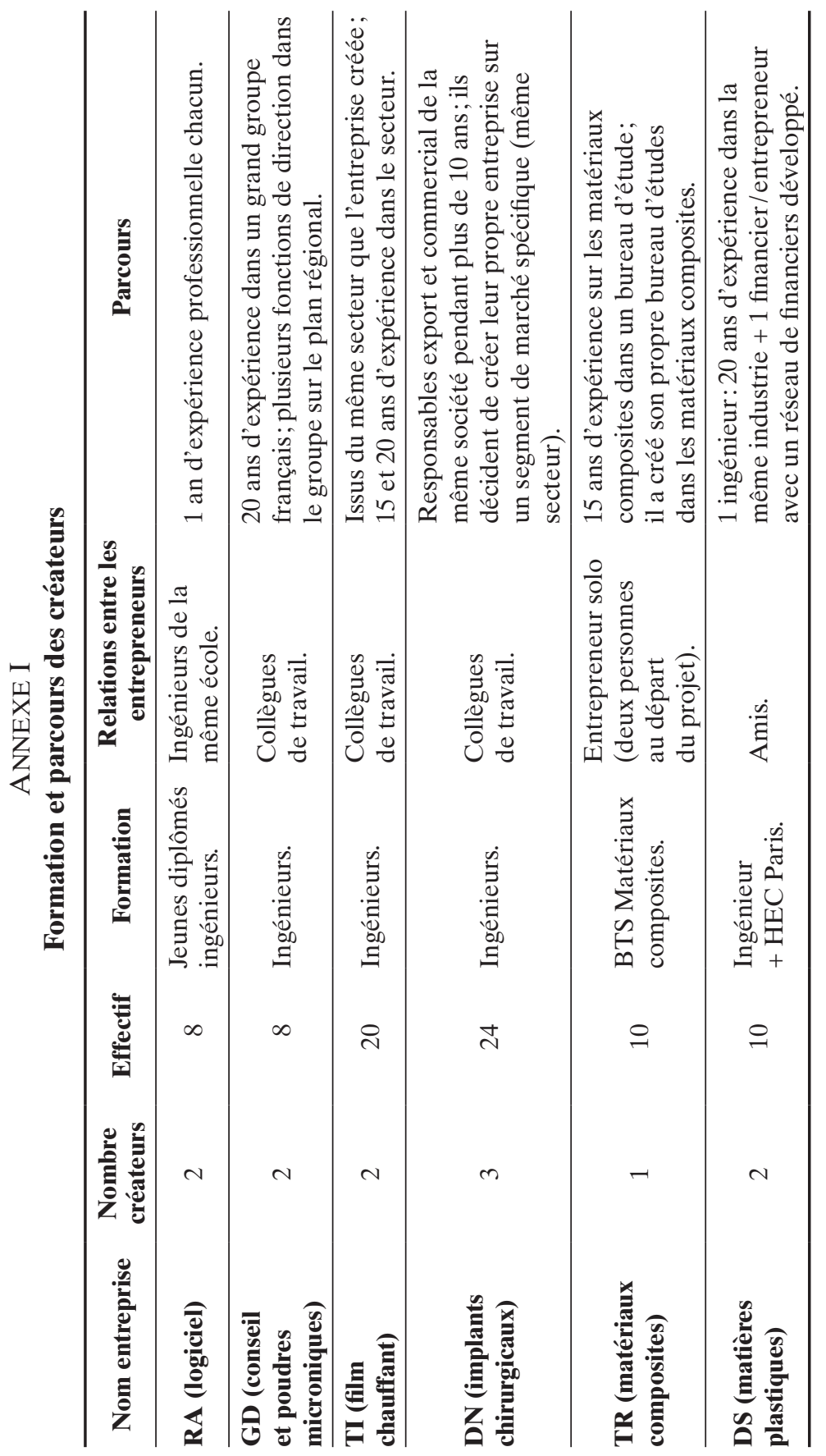




\section{Bibliographie}

AgEron, B. et I. HuAult (2002), «Complexité du processus d'internationalisation de la P.M.E: vers un enrichissement de l'analyse behavioriste», Management International, vol. $6, \mathrm{n}^{\circ}$ 2, p. 43-53.

ALDRICH, H. et C. ZIMMER (1986), «Entrepreneurship through social networks », dans D. Sexton et R.W. Smilor (dir.), Art and Science of Entrepreneurship, Cambridge, MA, Ballinger Publishing Company, p. 3-23.

ANDERSSON, S. et F. EVANGELISTA (2006), «The entrepreneur in the Born Global firm in Australia and Sweden », Journal of Small Business and Enterprise Development, vol. 13, n 4, p. 642-659.

AUTIO, E., H.J. SAPIENZA et G. ALMEIDA (2000), «Effects of age at entry, knowledge intensity and imitability on international growth», Academy of Management Journal, vol. 43, n 5, p. 902-906.

BALDEGGER, R.J. et P. SCHUEFFEL (2009), «Le comportement d'internationalisation des PME suisses: born global et internationalisation progressive», Revue internationale PME, vol. 22, $\mathrm{n}^{\circ}$ 1, p. 9-45.

BARNEY, J.B. (1991), «Firm resources and sustained competitive advantage », Journal of Management, vol. 17, $\mathrm{n}^{\circ}$ 1, p. 99-120.

Bell, J., R. MCNAUGHTON, S. YOUNG et D. CRICK (2003), «Towards an integrative model of small firm internationalization », Journal of International Entrepreneurship, vol. 1, p. 339-362.

CALOF, J.L. (1994), «The relationship between firm size and export behavior revisited», Journal of International Business Studies, vol. 25, n 2, p. 367-387.

CHETTY, S. et C. CAMPBELL-HUNT (2004), «A strategic approach to internationalization: a traditional versus a "born-global" approach », Journal of International Marketing, vol. $12, \mathrm{n}^{\circ} 1, \mathrm{p} .57-81$.

COVIELlO, N.E. (2006), «The network dynamics of international new ventures», Journal of International Business Studies, vol. 37, $\mathrm{n}^{\circ}$ 5, p. 713-731.

COVIELlO, N.E et A. MCAULEY (1999), «Internationalization and the smaller firm: a review of contemporary empirical research», Management International Review, vol. 39, n 3, p. 223-256.

COVIELlO, N.E. et H.J. MUNRO (1997), «Network relationships and the internationalisation process of small software firms », International Business Review, vol. $6, \mathrm{n}^{\circ}$ 4, p. 361-386.

DODD, S.D. (1997), «Social network membership and activity rates: some comparative data», International Small Business Journal, vol. 15, n 4, p. 80-87.

DUBINI, P. et H.ALDRICH (1991), «Personal and extended networks are central to the entrepreneurial process », Journal of Business Venturing, vol. 6, $\mathrm{n}^{\circ}$ 5, p. 305-313.

EISENHARDT, K.M. (1989), «Building theories from case study research », Academy of Management Review, vol. 14, $\mathrm{n}^{\circ}$ 4, p. 532-550. 
ELLIS, P. (2000), «Social ties and foreign market entry», Journal of International Business Studies, vol. 31, n 3, p. 443-469.

ELLIS, P. et A. PECOTICH (2001), «Social factors influencing export initiation in small and medium-sized enterprises », Journal of Marketing Research, vol. 38, $\mathrm{n}^{\circ} 1, \mathrm{p} .119-130$.

FAVRE-BONTÉ, V. et J.L. GIANNELLONI (2007), «L'influence des caractéristiques de personnalité du dirigeant de PME sur la performance à l'export», Actes de la $X V I^{e}$ conférence AIMS, Montréal, 6-9 juin.

GRANOVETTER, M. (1985), «Economic action and social structure: the problem of embeddedness », American Journal of Sociology, vol. 91, n 3, p. 481-510.

HARRIS, S. et C. WHEELER (2005), «Entrepreneurs' relationships for internationalization: functions, origins and strategies», International Business Review, vol. 14, $\mathrm{n}^{\circ}$ 2, p. 187-207.

JOHANNISSON, B. (1987), «Anarchists and organisers: entrepreneurs in a network perspective», International Studies of Management and Organisation, vol. 17, p. 49-63.

JOHANNISSON, B., K.N. ALEXANDERSON et K. SENNESETH (1994), «Beyond anarchy and organisation: entrepreneurs in contextual networks », Entrepreneurship and Regional Development, vol. 6, $\mathrm{n}^{\circ}$ 4, p. 329-356.

JOHANSON, J. et L.G. MATTSON (1988), «Internationalization in industrial systems: a network approach», dans N. Hood et J.E. Vahlne (dir.), Strategies in Global Competition, Londres, Croom Helm.

JOHANSON, J. et J.-E. VAHLNE (1990), «The mechanism of internationalization», International Marketing Review, vol. 7, $\mathrm{n}^{\circ}$ 4, p. 11-24.

MADSEN, T.K. et P. SERVAIS (1997), «The internationalisation of Born Global: an evolutionary process? », International Business Review, vol. 6, nº 6, p. 561-583.

MCDOUgALl, P.P., B. OVIATT et R.C. SHRADER (2003), «A comparison of international and domestic new ventures », Journal of International Entrepreneurship, vol. $1, \mathrm{n}^{\circ} 1$, p. 59-82.

MCDOUGALL, P.P., S. SHANE et B.M OVIATT (1994), «Explaining the formation of international new ventures: the limits of theories from international business research », Journal of Business Venturing, vol. 9, n 6, p. 469-487.

MCKINSEY \& CO. (1993), Emerging Exporters : Australia's High Value-added Manufacturing Exporters, Melbourne, Australian Manufacturing Council.

Miles, M. et A. Huberman (1994), Qualitative Data Analysis,2e éd., Thousand Oaks, Sage.

O’Donnel, A., A. Gilmore, D. Cummins et D. CARSON (2001), «The network construct in entrepreneurship research : a review and critique», Management Decision, vol. 39, n 9, p. 749-760.

OJALA, A. (2009), «Internationalization of knowledge-intensive SMEs: the role of network relationships in the entry to a psychically distant market », International Business Review, vol. 18, $\mathrm{n}^{\circ} 1$, p. 50-59. 
OSTGAARD, T.A. et S. BIRLEY (1994), «Personal networks and firm competitive strategy: a strategic or coincidental match?», Journal of Business Venturing, vol. $9, \mathrm{n}^{\circ} 4$, p. 281-305.

OVIATT, B.M. et P.P. MCDOUGALL (1994), «Toward a theory of international new ventures », Journal of International Business Studies, vol. 24, n 1, p. 45-64.

OviATT, B.M. et P.P. MCDOUGALL (1995), «Global start-ups : entrepreneurs on an worldwide stade», Academy of Management Executive, vol. 9, n² 2, p. 30-43.

OviATT, B.M. et P.P. MCDOUGALL (1997), «Challenges for internationalisation process theory: the case of international new ventures », vol. $37, \mathrm{n}^{\circ} 2$, p. 85-99.

REID, S.D. (1981), «The decision-maker and export entry and expansion», Journal of International Business Studies, vol. 12, n² 2, p. 101-112.

RIALP,A., J. RIALP et G.A. KNIGHT (2005), «The phenomenon of early internationalizing firms: what do we know after a decade (1993-2002) of scientific inquiry? », International Business Review, vol. 14, $\mathrm{n}^{\circ}$ 2, p. 147-166.

ROYER, I. et P. ZARLOWSKI (1999), «Le design de la recherche», dans R. A. Thiétart et al., Méthodes de recherche en management, Paris, Dunod, p. 139-168.

SERVAIS, P. et E.S. RASMUSSEN (2000), «Different types of international new ventures », Academy of International Business, novembre, Phoenix, p. 1-27.

SHANE, S. (2000), «Prior knowledge and the discovery of entrepreneurial opportunities », Organization Science, vol. 11, n 4, p. 448-469.

SHARMA, D.D. et A. BLOMSTERMO (2003), «The internationalization process of born global: a network view», International Business Review, 12, n 6, p. 730-753.

STAKE, R.E. (1994), «Case studies», dans N.K. Denzin et Y.S. Lincoln (dir.), Handbook of Qualitative Research, Thousand Oaks, CA, Sage, p. 236-247.

TAN, A., P. BREWER et P.W. LIESCH (2007), «Before the first export decision : internationalisation readiness in the pre-export phase », International Business Review, vol. 16, n 3, p. 294-309.

Weerawardena, J., G.S. Mort, P.W. Liesch et G. KNIGHT (2007), «Conceptualizing accelerated internationalization in the born global firm: a dynamic capabilities perspective », Journal of World Business, $\mathrm{n}^{\circ}$ 42, $\mathrm{n}^{\circ}$ 3, p. 294-306.

YIN, R.K. (1989), Case Study Research: Design and Methods, Beverly Hills, CA, Sage.

ZuCCHElla, A., G. PAlAmARA et S. DeniCOlAi (2007), «The drivers of the early internationalization of the firm », Journal of World Business, vol. 42, $\mathrm{n}^{\circ} 3$, p. 268-280. 